\title{
Contrast mechanisms in high-resolution contact lithography: A comparative study
}

\author{
Michael Paulus $^{\mathrm{a}, \mathrm{b}, *}$, Heinz Schmid ${ }^{\mathrm{b}}$, Bruno Michel $^{\mathrm{b}}$, Olivier J.F. Martin ${ }^{\mathrm{a}}$ \\ ${ }^{a}$ Electromagnetic Fields and Microwave Electronics Laboratory, Swiss Federal Institute of Technology, ETH-Zentrum, \\ ETZ, CH-8092 Zurich, Switzerland \\ ${ }^{\mathrm{b}}$ IBM Research, Zurich Research Laboratory, CH-8803 Rüschlikon, Switzerland
}

\begin{abstract}
We compare three different approaches to high-resolution contact lithography with special emphasis on contrast mechanisms for subwavelength structures. Masks with protruding metal absorbers, masks with absorbers embedded in the transparent background, and masks with air gaps and recessed absorbers are studied. Using the Green's tensor technique we compute the light intensity distribution in the photoresist. The intensity and contrast functions are investigated for different mask geometries (absorber thickness, height of protruding elements), and the difference between chrome and gold as absorber material is discussed. Our results show that embedding the absorbers in a transparent mask material enhances the transmitted intensity and the contrast compared with a mask having protruding metal absorbers. A further improvement is achieved by a topographically patterned mask with air gaps and recessed absorbers. Optimized mask dimensions can be found for which the contrast and the depth of focus are increased. (c) 2001 Elsevier Science B.V. All rights reserved.
\end{abstract}

Keywords: Contact lithography; Contrast mechanism; Electric field distribution; Scattering calculation; Green's tensor technique

PACS: 07.05.Tp; 42.25.-p; 85.40.Hp

\section{Introduction}

In recent years, research interest has been revived in utilizing the advantages of optical contact lithography for replicating structures in the subwavelength regime. There are several approaches to this task: high-resolution contact masks with protruding metal absorbers (metal-protruding masks, MPM) [1,2], masks where the absorbers are embedded in the transparent background material (metal-embedded masks, MEM) [3,4], topographically patterned masks with air gaps and recessed absorbers (light-coupling masks, LCM) [5], and structured masks where no absorber is used (phase masks) [6]. Whereas the latter approach is based on interferences between light passing through

\footnotetext{
*Corresponding author.

E-mail address: paulus@ifh.ee.ethz.ch (M. Paulus).
} 
materials with different optical thicknesses, the first three techniques use an amplitude contrast between transparent and opaque parts of the mask. These mask concepts have in common that they rely on a flexible glass or polymer, which allows the mask to be placed in contact with the entire substrate. This is the most important requirement for reliable subwavelength contact lithography.

With these techniques the formation of features in the 100-nm range was successfully demonstrated. Several theoretical studies have been published to investigate the potentials of these approaches for subwavelength patterning and to determine the corresponding optical interactions in the mask [7,8]. However, it has not yet been studied how these concepts compare to each other and whether one approach provides better performance. In this article we focus on the three types of amplitude masks (MPM, MEM, LCM) and investigate their optical properties. Fig. 1 shows a schematic view of these mask types. Whereas in an MEM and an LCM the light travels through the homogeneous transparent background material into the photoresist, in an MPM it has to cross the air gap between the mask substrate and the photoresist. It is assumed that further experimental parameters (e.g. contact with the photoresist surface, resist response, treatment after the exposure) are identical for all three systems.

\section{Model}

For the calculation of the electric field distribution $\boldsymbol{E}$ we used a two-dimensional, self-consistent numerical approach based on the Green's tensor technique [9]. This fully vectorial technique is based on the electric field integral equation and enables the investigation of the scattering and propagation of light through the mask/photoresist system. The advantage of this approach lies in the fact that the

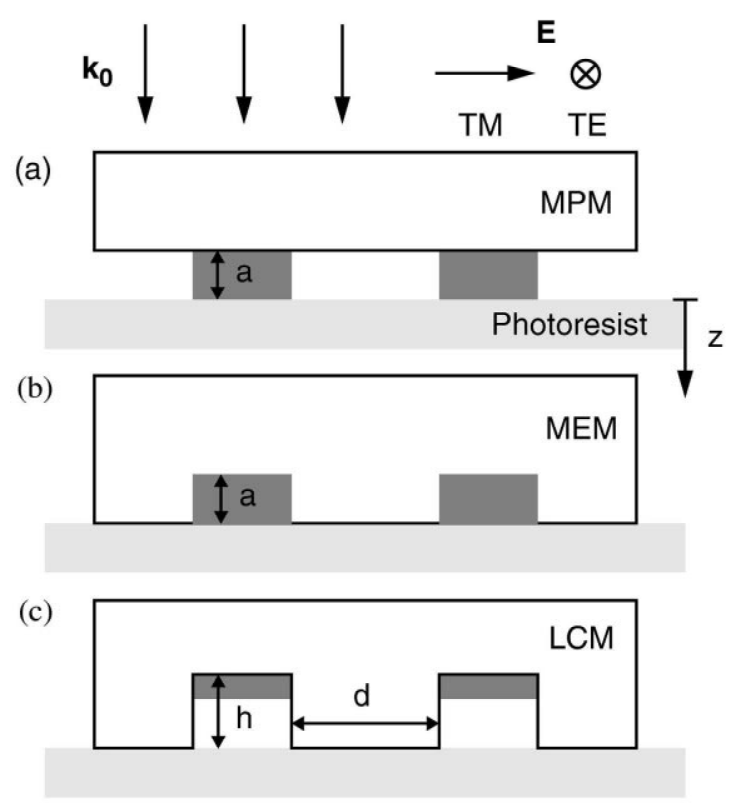

Fig. 1. Schematic view of the compared masks for contact lithography: (a) metal-protruding mask (MPM); (b) metalembedded mask (MEM); (c) light-coupling mask (LCM). 
boundary conditions at the interfaces between different materials, as well as at the edges of the computation window are perfectly and automatically fulfilled.

For our study we consider isolated lines with a line width of $d=100 \mathrm{~nm}$ and as absorber material in the mask we chose chrome or gold because these metals are most frequently used in experiments. The determinant parameter to characterize a given exposure is the intensity $I=\boldsymbol{E} \cdot \boldsymbol{E} *$. The results presented here refer to a plane-wave illumination perpendicular to the mask surface with a vacuum wavelength of $248 \mathrm{~nm}$. The intensity of the incident field is normalized to unity, and a circular polarization is used for all computations if not stated otherwise. The optical properties of the simulated material systems are assumed to be linear and isotropic. The relative permittivities at this wavelength are $\epsilon=2.25$ for the mask background, $\epsilon=2.5$ for the photoresist, $\epsilon=-3.4+i 3.6$ for chrome, and $\epsilon=-0.9+i 4.3$ for gold [10].

\section{Results}

We first compare the contrast that can be achieved by the different techniques. For this purpose we define the contrast $C$ as

$C=\frac{I_{\max }-I_{0}}{I_{\max }+I_{0}}$

where $I_{\max }$ is the maximal intensity of the transmitted light and $I_{0}$ is the intensity of the light that leaks through the absorbers and forms the background for the exposure.

In Fig. 2 we compare MEMs and MPMs with chrome or gold absorbers as a function of the absorber thickness $a$. All curves show a strongly enhanced contrast with increasing absorber thickness, because the intensity $I_{0}$ of the light leaking through the absorbers decreases exponentially.

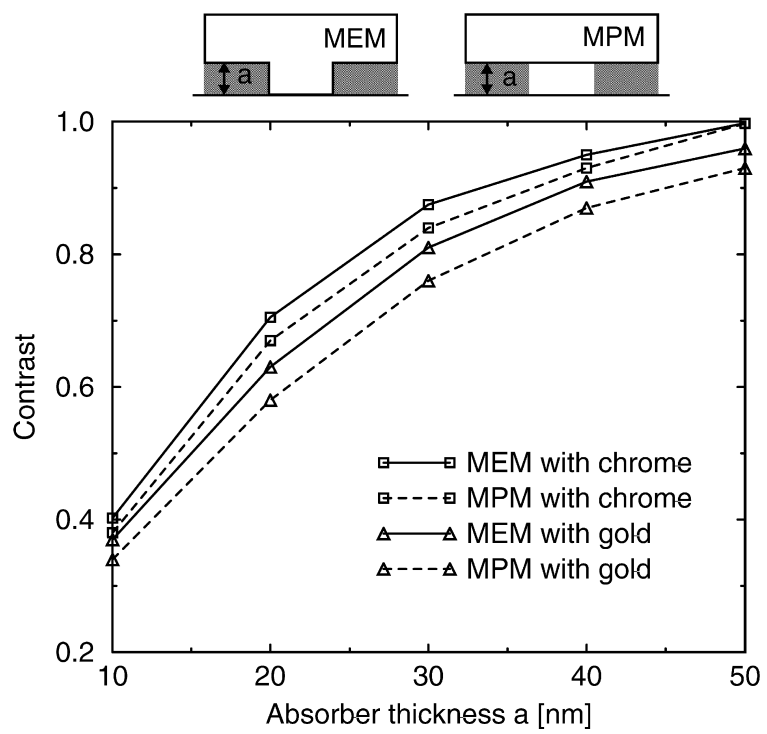

Fig. 2. Contrast as defined in Eq. (1) of MEMs and MPMs as a function of absorber thickness $a$ at $z=10 \mathrm{~nm}$. Chrome or gold is used as absorber material. 


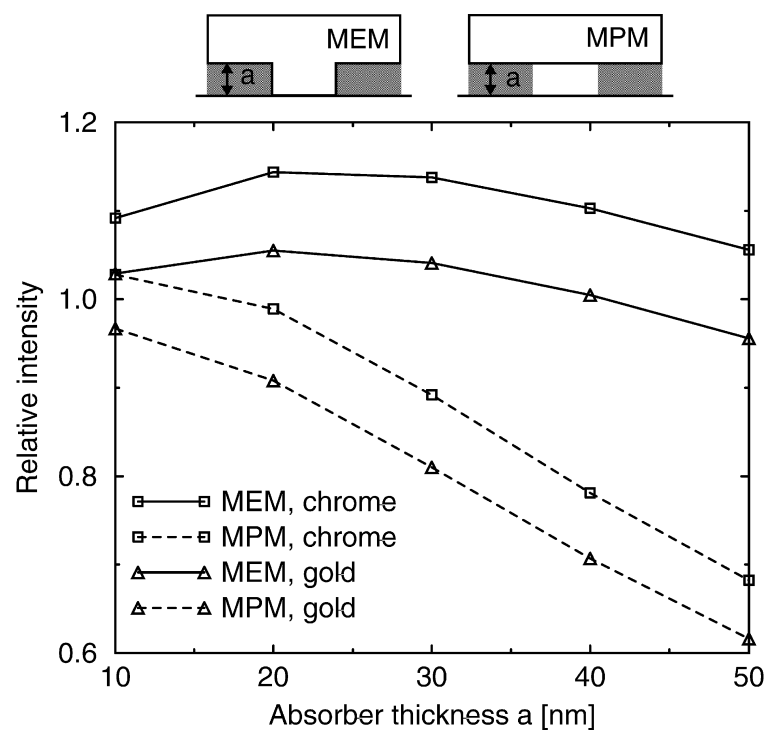

Fig. 3. Maximal intensity $I_{\max }$ as a function of absorber thickness $a$ at $z=10 \mathrm{~nm}$. MEMs and MPMs are compared using chrome or gold as absorber material.

In the limit of a thick absorber, $C$ converges to 1 for all curves because the background $I_{0}$ in Eq. (1) becomes 0 . Furthermore, the contrast of the chrome masks is greater than the contrast of the gold masks. The higher absorbance and higher reflectivity of chrome leads not only to a smaller background at the shadowed regions but also to a stronger field enhancement in the opening and, consequently, to a larger transmitted intensity. This fact is proved by Fig. 3, where the intensity $I_{\max }$ for MEMs and MPMs is reported for different absorber thicknesses.

Figs. 2 and 3 also show that the contrast and the intensity of the MEM are slightly superior to those of the MPM, because reflections at the two interfaces mask substrate/air and air/photoresist lower the transmitted intensity [33\% for the worst case of a $\lambda / 4=62-\mathrm{nm}$ thick air slab (not shown) and by $16 \%$ for a 30-nm thick air slab, Fig. 3].

Note that the intensities for the MEMs have a maximum at $a=20 \mathrm{~nm}$, which represents the tradeoff between two mechanisms: The transmitted intensity decreases, whereas the confinement of light becomes more efficient with increasing metal thickness.

The contrast increases similarly if we fill metal into the air gaps of an LCM with a fixed total protrusion height $h$, because the absorber significantly lowers the background $I_{0}$. However, as a thicker metal also lowers the intensity transmitted into the resist, there is a tradeoff between good contrast and enough light passing through the mask. In the following we shall consider an LCM with a constant metal thickness of $a=20 \mathrm{~nm}$ (corresponding to the optimum in Fig. 3) and study the influence of the lateral air gaps. Fig. 4 reports the contrast of LCMs with chrome or gold absorbers as a function of the total protrusion height $h$. Note that the total protrusion height $h$ includes both the absorber thickness $a$ and the remaining air gap. Again, chrome proves to be the metal that produces higher contrast. In Fig. 4 we also notice that $C$ reaches a maximum at $h=75 \mathrm{~nm}$. This maximum corresponds to the maximal reflectivity of the metal/air slab, leading to a minimum of $I_{0}$. Thus, by choosing the appropriate total protrusion height, the contrast can be increased by $15 \%$ for a gold absorber and by $10 \%$ for a chrome absorber. As this effect is solely related to the air gap of the LCM, 


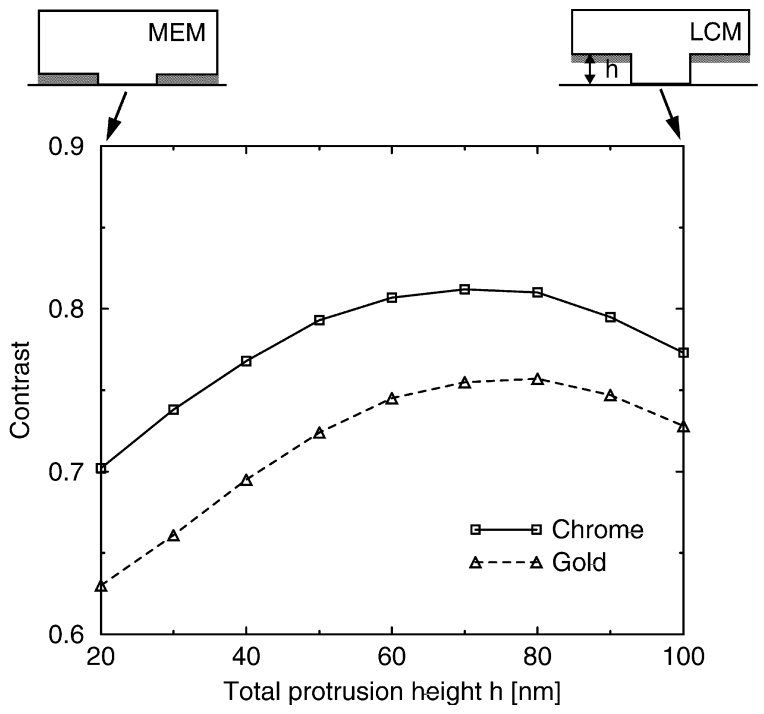

Fig. 4. Contrast of LCMs as a function of the total protrusion height $h$ at $z=10 \mathrm{~nm}$. All masks have a 20-nm thick chrome or gold absorber. The leftmost values correspond to an MEM with $a=20 \mathrm{~nm}$.

it is independent of the absorber thickness $a$. For a thicker absorber (leading to a reduction of the background), a similar effect is observed and an optimal total protrusion height $h$ can be found.

The intensity distribution should ideally show not only a large difference between the regions to be illuminated and the shadowed regions, but also a shape that accurately replicates the pattern in the resist. To assess this requirement as a function of the additional air gap we computed the electric field at different depths in the photoresist, $z=10 \mathrm{~nm}, z=100 \mathrm{~nm}$ and $z=250 \mathrm{~nm}$, and determined the full width at half maximum (FWHM) of the intensity distribution. Fig. 5 presents line cuts through the

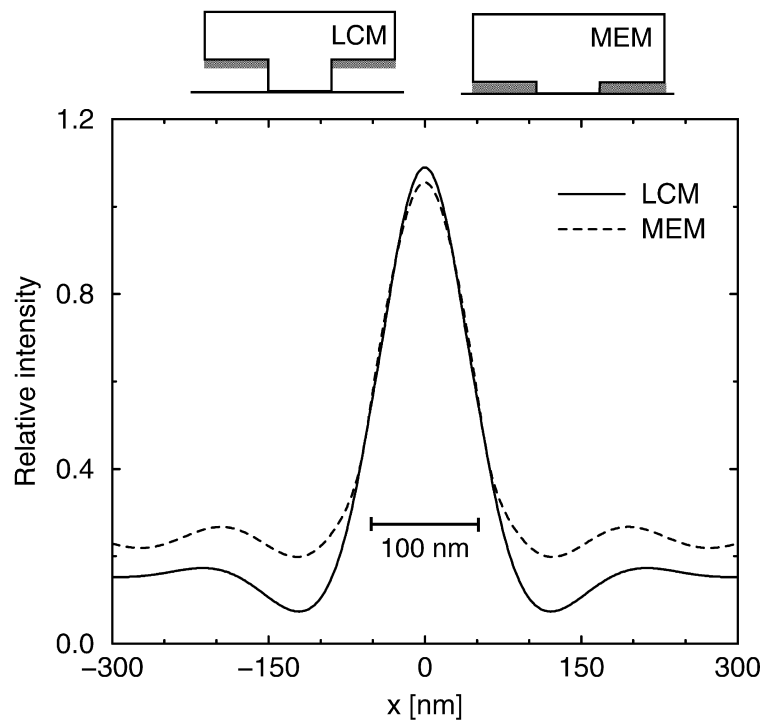

Fig. 5. Relative intensity distributions at $z=10 \mathrm{~nm}$ for an MEM and an LCM. Both masks have a 20-nm thick gold absorber; the total protrusion height of the LCM is $h=80 \mathrm{~nm}$. The bar represents the original line width. 
intensity distribution close to the mask $(z=10 \mathrm{~nm})$ for an LCM and an MEM. The masks have a 20-nm thick gold absorber, the total protrusion height $h$ of the LCM is $80 \mathrm{~nm}$, which is close to the optimum in Fig. 4. The FWHM of both line cuts is $\approx 90 \mathrm{~nm}$, and the intensity at the edges of the mask has dropped to $I=0.6$. With the additional air gap of the LCM, however, the confinement of the transmitted light is improved, leading to a slightly greater maximal intensity. This effect is even more pronounced for the intensity distribution deeper in the photoresist. Fig. 6 shows line cuts of 100 and $250 \mathrm{~nm}$ depth in the photoresist. The shape of the intensity distributions becomes substantially more narrow and higher for the LCM. The FWHM decreases by $\approx 10 \%$ at $z=100 \mathrm{~nm}$ and even by $\approx 20 \%$ at $z=250 \mathrm{~nm}$, compared to MEM. Concurrently, at both depths the peak intensity is $10 \%$ greater for LCM than for MEM. The air gap in the LCM improves the guiding of the light into the resist, resulting in greater directionality without the loss of intensity that would occur with thick full-metal absorbers. At the corners of the targeted line, $100 \mathrm{~nm}$ deep in the photoresist, the relative intensity is $I=0.6$ for both masks. This is still close to the value at $z=10 \mathrm{~nm}$ (Fig. 5) providing steep edges of the developed resist. At $z=250 \mathrm{~nm}$ the targeted line is defined only by the $I=0.5$ isointensity lines for both masks. Hence, this corresponds to the bottom region of the developed photoresist.

If we compare the intensity distributions of MEMs with MPMs having an identical absorber, the FWHMs of the MPMs are $14 \%$ greater close to the mask $(z=10 \mathrm{~nm})$ and $17 \%$ greater deeper in the photoresist $(z=100 \mathrm{~nm})$. Hence, not only a smaller portion of the illuminating light is transmitted, but the directionality provided by the mask opening is less efficient.

Until now computations were carried out for circularly polarized illumination. To show in more detail the influence of the mask composition on polarization, Fig. 7 reports the intensity distributions when a TE or TM-polarized illumination is used (see Fig. 1 for the definition of TE and TM). Again, an MEM with a 20-nm gold absorber is compared with an LCM with $20 \mathrm{~nm}$ of gold and a 60-nm air gap (total protrusion height $h=80 \mathrm{~nm}$ ). For both polarizations the LCM shows a slightly higher transmitted intensity. Owing to the orientation of the TE-polarized electric field vector the intensity is focused on the center of the opening, resulting in a narrow peak with an FWHM of $\approx 80 \mathrm{~nm}$ for both masks. In contrast, the TM electric field vector is perpendicular to the surfaces, and hence the

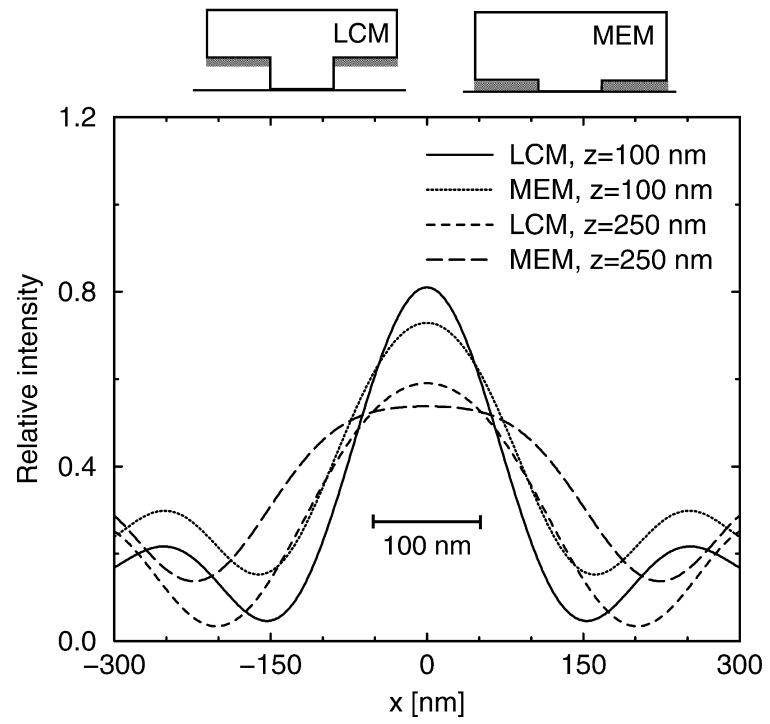

Fig. 6. Same as Fig. 5, but at a depth of $z=100$ and $250 \mathrm{~nm}$. 


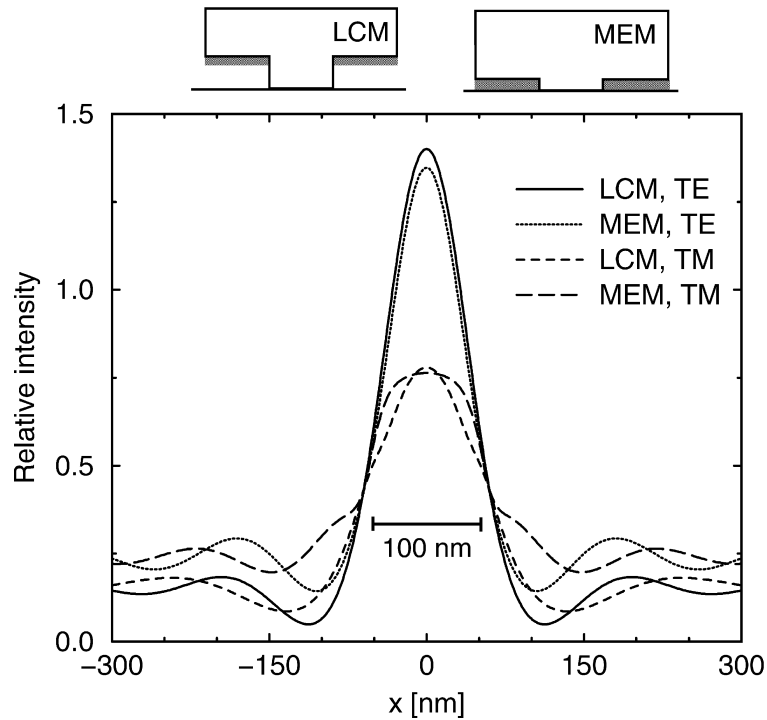

Fig. 7. Polarization-dependent relative intensity distributions for an MEM and an LCM at $z=10 \mathrm{~nm}$. TE or TM-polarized light is used as illumination (see Fig. 1). Both masks have a 20-nm thick gold absorber; the total protrusion height of the LCM is $h=80 \mathrm{~nm}$. The bar represents the original line width.

intensity distribution is wider with an FWHM of $\approx 110 \mathrm{~nm}$ for both masks. This broadening is based on the continuity of the displacement field $D=\epsilon E$, resulting in a high intensity at the edges of the opening. Furthermore, the electric field is extremely enhanced at the metal corners, leading to the broader intensity distribution of the MEM. This effect also depends on the absorber metal and can be enhanced when surface plasmons are excited in the metal.

Similar results are obtained for an MPM, where the FWHM is about 5\% greater for both polarizations. However, in agreement with Fig. 3, the maximum peak values $I_{\max }$ of the relative intensity decrease significantly to 1.07 and 0.74 , respectively, for the TE and TM polarizations, respectively. Note that the dimensions used together with gold as absorber are not appropriate for a polarizing functionality.

\section{Discussion}

Following the laws of image formation in a lens system, the ultimate resolution $\Delta$ for pattern replication with optical lithography is given by the diffraction limit [11]:

$\Delta \approx a \frac{\lambda_{0}}{\mathrm{NA}}$

where $a$ is a process parameter, $\lambda_{0}$ the vacuum wavelength and NA the numerical aperture. Whereas for projection lithography this relation restricts the ultimate resolution to $\Delta \approx \lambda_{0} / 2$, for contact lithography this limit is further reduced. Since the refractive index $n$ of the mask lowers the effective wavelength $\lambda_{\text {eff }}$, structures with a size $\Delta \approx \lambda_{\text {eff }} / 2=\lambda_{0} /(2 n)$ can be realized. With the parameters used in this study (see Section 2) the resolution is enhanced to $\Delta \approx 248 /(2 \cdot 1.5) \mathrm{nm}=80 \mathrm{~nm}$. However, 
even smaller structures can be replicated with the evanescent near field created by each opening in the mask [7].

All three considered mask types are capable of subwavelength resolution. However, the different contrast mechanisms imply different intensity distributions in the photoresist during exposure. For MEMs and MPMs a thicker metal absorber leads to an enhanced contrast (Fig. 2), but also lowers the intensity in the photoresist (Fig. 3) and, consequently, requires a more costly experimental setup. Especially with MPMs the amount of transmitted light is strongly reduced. The contrast can be further increased by an additional air gap below the metal absorbers (LCMs, Fig. 5). Since these air gaps improve the directional guiding of light, the intensity distribution remains better confined even deeper inside of the photoresist.

\section{Summary}

We used simulations based on the Green's tensor technique to precisely model and optimize the design of high-resolution contact lithography masks. Our calculations show that chrome is a superior absorber to gold because of its greater reflectivity and absorbance. We compared three different approaches to high-resolution contact lithography and studied the contrast mechanisms. We showed that embedding the absorbers in a transparent background material increases the transmitted light and enhances the contrast. An even further improvement at fixed metal thickness is achieved if topographically patterned masks are used, where the absorbers are recessed and the lateral air gaps lead to an enhanced confinement of the light.

\section{Acknowledgements}

The authors thank P. Seidler (IBM) and R. Vahldieck (ETH) for their support of the project and gratefully acknowledge the funding of the Swiss National Science Foundation.

\section{References}

[1] H.I. Smith, Method for fabricating high frequency surface wave transducers, Rev. Sci. Instrum. 40 (1969) $729-730$.

[2] M.M. Alkaisi, R.J. Blaikie, S.J. McNab, R. Cheung, D.R.S. Cumming, Sub-diffraction-limited patterning using evanescent near-field optical lithography, Appl. Phys. Lett. 75 (22) (1999) 3560-3562.

[3] U.C. Fischer, H.P. Zingsheim, Submicroscopic pattern replication with visible light, J. Vac. Sci. Technol. 19 (4) (1981) $881-885$.

[4] J.G. Goodberlet, Patterning $100 \mathrm{~nm}$ features using deep-ultraviolet contact photolithography, Appl. Phys. Lett. 76 (6) (2000) 667-669.

[5] H. Schmid, H. Biebuyck, B. Michel, O.J.F. Martin, Light-coupling masks for lensless, sub-wavelength optical lithography, Appl. Phys. Lett. 72 (1998) 2379-2381.

[6] J.A. Rodgers, K.E. Paul, R.J. Jackman, G.M. Whitesides, Using an elastomeric phase mask for sub-100 nm photolithography in the optical near field, Appl. Phys. Lett. 70 (20) (1997) 2658-2660.

[7] S.J. McNab, R.J. Blaikie, Contrast in the evanescent near field of $\lambda / 20$ period gratings for photolithography, Appl. Opt. 39 (1) (2000) 20-25.

[8] O.J.F. Martin, N.B. Piller, H. Schmid, H. Biebuyck, B. Michel, Energy flow in light-coupling masks for lensless optical lithography, Optics Express 3 (1998) 280-285.

[9] M. Paulus, O.J.F. Martin, Light propagation and scattering in stratified media: A. Green's tensor approach, J. Opt. Soc. Am. A 18 (4) (2001) 854-861.

[10] O.S. Am, 2nd Edition, Handbook of Optics, Vol. II, McGraw-Hill, New York, 1995.

[11] M. Born, E. Wolf, Principles of Optics, 6th Edition, Pergamon Press, Oxford, 1987. 